\title{
Effect of Hand Massage on Occupational Leg Swelling and Resting-state Electroencephalographic Activity: A Randomized Cross-over Study
}

Hideki Nakano $^{{ }^{*}}$, Takayuki Kodama ${ }^{1}$, Masashi Sakamoto ${ }^{2}$, Tomohiro Ueda ${ }^{2}$, Tomiko Tani ${ }^{3,4}$, Ikuko Mori ${ }^{3,4}$ and Shin Murata ${ }^{1}$

${ }^{1}$ Department of Physical Therapy, Faculty of Health Science, Kyoto Tachibana University, Kyoto-city, Kyoto, Japan

${ }^{2}$ Graduate School of Health Science, Kyoto Tachibana University, Kyoto-city, Kyoto, Japan

${ }^{3}$ Naris Cosmetics Company Limited, Osaka-city, Osaka, Japan

${ }^{4}$ Japan Wellness Therapist Association, Osaka-city, Osaka, Japan

\section{Abstract}

Background: Hand massage is recommended for leg swelling with circulatory disturbance because sitting or standing for hours leads to occupational leg swelling and increases the risk for development of chronic venous disease. Furthermore, studies have revealed that hand massage improves emotion and mood. Hence, this study aimed to investigate the effects of hand massage on occupational leg swelling and resting-state electroencephalographic (EEG) activity.

Methods: This study was designed as a randomized cross-over study. Eighteen healthy women who work in a sitting or standing position were included in this study and were randomly divided into 2 groups (A and B). First, group A received hand massage to their right leg, and group B received machine massage to their right leg, for 15 minutes each. After 2 weeks, group A received machine massage to their right leg, and group B received hand massage to their right leg, for 15 minutes each. Lower leg volume, restingstate EEG activity were measured before and after each massage. Moreover, the emotion and mood states were measured after each massage.

Results: Lower leg volume was significantly reduced after hand and machine massages $(p<0.05)$. The degree of pleasant, relaxation and refreshing was significantly higher after hand massage than after machine massage $(\mathrm{p}<0.05)$. Resting-state alpha activity after hand massage significantly increased in the left anterior cingulate cortex compared with resting-state alpha activity after machine massage $(\mathrm{p}<0.05)$.

\section{Publication History:}

Received: May 23, 2018

Accepted: June 13, 2018

Published: June 15, 2018

\section{Keywords:}

Alpha wave, Anterior cingulate cortex, Emotion, Exact lowresolution brain Electromagnetic tomography, Hand massage, Lower leg volume, Mood, Occupational leg swelling, Randomized cross-over study, Resting-state electroencephalograph

Conclusion: This study suggests that hand massage attenuate occupational leg swelling and increases the resting-state alpha activity in the left anterior cingulate cortex.

\section{Introduction}

Occupational leg symptoms, such as swelling, pain, and feeling of heaviness and tension in the legs frequently occur in healthy individuals who work for hours in the sitting or standing positions [1]. Sitting or standing for hours decreases the blood flow out of the legs and results in increased pressure on the veins; therefore, prolonged sitting and standing are both risk factors for the development of chronic venous disease [2]. Occupational leg symptoms, especially leg swelling is associated with feelings of tiredness and heaviness of the legs, and leg pain $[1,3,4]$; therefore, reducing leg swelling is an important approach to prevent the development of the chronic venous disease.

Previous studies have reported that compression stocking [1,4,5-18], neuromuscular electrical stimulation device [8], and leg movement $[9,10]$ are effective in reducing the occupational leg swelling. These methods promote calf muscle pump efficacy, peak venous velocity, and venous return, thereby reducing ambulatory venous pressures, interstitial fluid volume, and leg volume $[5,11$ 13]. However, the above methods have disadvantages and cannot be applied to participants who have movement and sensory disorders, circulatory disturbance, and inflammation of the skin. On the other hand, it is reported that hand massage is effective in reducing chronic edema and lymphedema [14,15], and leg edema during pregnancy and postpartum $[16,17]$. Hand massage is advantageous in that it can be performed on a participant with the above-mentioned disorders because the intensity and treatment site can be manipulated manually. However, the effect of hand massage on occupational leg swelling has not been clarified. In addition, studies have revealed that hand massage improves emotion and mood [18,19]; however, the underlying neural mechanism has not been sufficiently verified. Hence, this study aimed to investigate the effect of hand massage on occupational leg swelling and resting-state electroencephalographic (EEG) activity.

\section{Materials and methods}

\section{Participants}

Eighteen healthy women (mean age \pm standard deviation: $47.5 \pm$ 16.2 years; mean height: $158.0 \pm 4.4 \mathrm{~cm}$; mean bodyweight: $50.9 \pm$ $4.9 \mathrm{~kg}$ ) who work in a sitting or standing position were included in this study. Only women were recruited because women have a higher incidence rate of leg swelling [1]. Participants with Mini-Mental State Examination (MMSE) scores below 24, cardiovascular or orthopedic diseases that might influence the results were excluded.

The study was conducted according to the principles of the Declaration of Helsinki and was approved by the local Institutional Ethics Committee (Kyoto Tachibana University). All subjects provided written informed consent and were free to withdraw from the study at any time.

"Corresponding Author: Dr. Hideki Nakano, Department of Physical Therapy, Faculty of Health Science, Kyoto Tachibana University, 34 Yamada-cho, Oyake Yamashina-ku, Kyoto-city, Kyoto 607-8175, Japan, Tel: +81-75-571-1111, Fax: +81-75-574-4122; E-mail: nakano.neuroreha@gmail.com

Citation: Nakano H, Kodama T, Sakamoto M, Ueda T, Tani T, et al. (2018) Effect of Hand Massage on Occupational Leg Swelling and Resting-state Electroencephalographic Activity: A Randomized Cross-over Study. Int J Clin Res Trials 3: 125. doi: https://doi.org/10.15344/2456-8007/2018/125

Copyright: (C) 2018 Nakano et al. This is an open-access article distributed under the terms of the Creative Commons Attribution License, which permits unrestricted use, distribution, and reproduction in any medium, provided the original author and source are credited. 
Citation: Nakano H, Kodama T, Sakamoto M, Ueda T, Tani T, et al. (2018) Effect of Hand Massage on Occupational Leg Swelling and Resting-state Electroencephalographic Activity: A Randomized Cross-over Study. Int J Clin Res Trials 3: 125. doi: https://doi.org/10.15344/2456-8007/2018/125

Page 2 of 5

\section{Study Protocol}

This study was designed as a randomized cross-over study (Figure 1). First, the participants were divided into 2 groups (A and B). Group $A$ received hand massage to their right leg and group $B$ received machine massage to their right leg, for 15 minutes each. After 2 weeks, group A received machine massage to their right leg and group B received hand massage to their right leg, for 15 minutes each.

The participants were seated comfortably in a chair with back and armrests and then received either a hand or machine massage. The massaged right lower limb was held at 30 degrees of knee flexion. Hand massage was performed by a therapist who had worked as an instructor at the Japan Wellness Therapist Association for 5 years. Massage oil (2E32000, Naris Cosmetics Co., LTD, Japan) was used during the hand massage to prevent friction and discomfort. A standard massage technique, without pressure on the points indicated in foot reflexology [21], was used in this study. First, the therapist stroked the entire lower leg, from the ankle to the knee, using her entire hand ( 5 minutes). Next, she stroked the entire foot from the toes to the ankle ( 5 minutes). Further, she stroked the toes one by one from the base of the toes toward the tip ( 5 minutes). The same therapist massaged all participants and was requested to keep the depth and rate of massage as consistent as possible. Machine massage was performed using the Foot Air Massager (HM-261, Omron Co. Ltd., Japan). This machine massaged, using pressurization, the toe, ankle, and the lower leg. Hand and machine massages were conducted for 15 minutes each. Lower leg volume and resting-state EEG activity were measured before (Pre1 and Pre2) and after (Post1 and Post2) each massage. Moreover, the emotion and mood states were measured after each massage (Post1 and Post2).

\section{Experimental measurements}

Lower leg volume was measured using the water displacement method. The reliability of the water replacement method has been reported in a previous study [22]. For the measurement, a rectangular shaped aquarium, with walls made of an acrylic board (length 300 $\mathrm{mm} \times$ width $150 \mathrm{~mm} \times$ height $450 \mathrm{~mm}$, Natsume Seisakusho Co., Ltd., Japan), was used. A drainage outlet was attached to the upper end of the aquarium, and any water overflow was discharged from the drainage outlet. Next, the participants gradually lowered their right lower limb into the full water tank, and the displaced, overflowing water was measured with a measuring cylinder.

The Likert scale [23] was utilized to measure the degree of pleasant, relaxation and refreshing, after each massage, to assess the emotional state and mood after each massage. The Likertscale consisted of a 5 -point scale ranging from 1 (strongly disagree) to 5 (strongly agree), and the higher scores indicated positive emotion and mood states.

EEG was obtained using a bio-signal recording system (Livo, Tec GihanCo., Ltd., Japan) and an active dry electrode system (g.SAHARA, g.tec Co., Ltd., Austria). EEG was recorded using 15 channels (Fpz, Fz, Cz, Pz, Oz, F3, F4, C3, C4, P3, P4, F7, F8, T7, T8) based on the international 10-20 system, at a sampling rate of 1000 $\mathrm{Hz}$. Reference electrodes were attached to both the earlobes. The recorded EEG data were processed as follows: down sampling to 512 $\mathrm{Hz}$, band pass filter set to $1-30 \mathrm{~Hz}$, independent component analysis to remove artifacts, and re-referencing to the average reference, using EEGLAB (Version 15) [24]. Next, the exact low-resolution brain electromagnetic tomography (eLORETA) was used to analyze the cortical distribution of current source density [25]. In the eLORETA, the solution space consists of 6239 cortical gray matter voxels at $5 \mathrm{~mm}$ spatial resolution, in a realistic head model [26], using the Montreal Neurological Institute (MNI) 152 template [27]. The eLORETA image was calculated in the alpha frequency band $(8-13 \mathrm{~Hz})$ because the resting alpha activity reflects the emotion and mood states [28].

\section{Statistical analysis}

Lower leg volume after each massage was analyzed with a 2 (massage: hand, machine) $\times 2$ (time: pre, post) ANOVA. We assessed whether massage or time showed any significant differences using the post hoc Bonferroni test. The degree of pleasant, relaxation, and refreshing after each massage was analyzed using the Wilcoxon signed-rank test. Statistical analyses were performed with SPSS ver. 24.0 (IBM, Chicago, IL, USA). The comparison of the cortical distribution of current source density, between massages in each frequency band, was assessed by the voxel-by-voxel independent sample F-ratiotests, based on the eLORETA log-transformed current density power. In the resulting statistical three-dimensional images, cortical

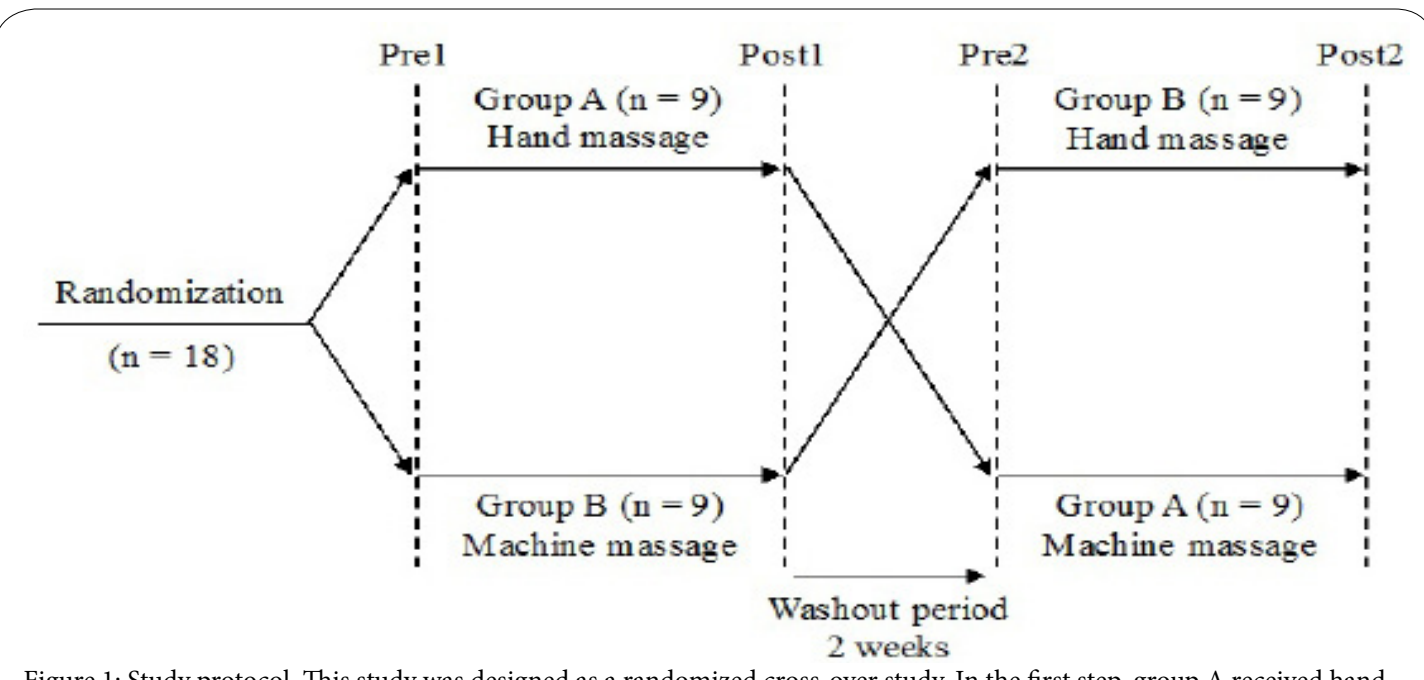

Figure 1: Study protocol. This study was designed as a randomized cross-over study. In the first step, group A received hand massage and group B received machine massage. After 2 weeks, group A received machine massage and group B received hand massage. Measures were assessed before (Pre1, Pre2) and after (Post1, Post2) each massage. 
Citation: Nakano H, Kodama T, Sakamoto M, Ueda T, Tani T, et al. (2018) Effect of Hand Massage on Occupational Leg Swelling and Resting-state Electroencephalographic Activity: A Randomized Cross-over Study. Int J Clin Res Trials 3: 125. doi: https://doi.org/10.15344/2456-8007/2018/125

Page 3 of 5

voxels exhibiting significant differences were identified utilizing a nonparametric approach (statistical nonparametric mapping; SnPM). The level of significance was set at a $\mathrm{p}<0.05$.

\section{Results}

Two-way ANOVA showed that there was a significant time effect, although there was no significant group $\times$ time interaction $(\mathrm{p}<0.05)$. Post-hoc Bonferroni comparisons revealed that the lower leg volume was significantly reduced after each massage compared with the lower leg volume before each massage $(\mathrm{p}<0.05$, Table 1$)$. In addition, the magnitude of change in lower leg volume before and after each massage (pre-value minus post-value) was $38.3 \pm 47.6 \mathrm{ml}$ for hand massage; and $45.6 \pm 56.3 \mathrm{ml}$ for machine massage. The degree of pleasant, relaxation and refreshing was significantly higher after hand massage than after machine massage $(p<0.05$, Table 2$)$. There was no significant difference observed between the resting-state alpha activity for pre-hand and pre-machine massage ( $p>0.05)$. Interestingly, the resting-state alpha activity after hand massage was significantly increased in the left anterior cingulate cortex compared with the resting-state alpha activity after machine massage $(\mathrm{p}<0.05)$ (Figure 2, Table 3).

\begin{tabular}{|c|c|c|c|c|c|c|c|c|c|}
\hline & \multicolumn{3}{|l|}{ Pre } & \multicolumn{3}{|l|}{ Post } & \multicolumn{3}{|c|}{ 1. Massage $\times$ Time } \\
\hline & \multirow[b]{2}{*}{ Mean } & & \multirow[b]{2}{*}{ SD } & \multirow[b]{2}{*}{ Mean } & & \multirow[b]{2}{*}{ SD } & \multicolumn{2}{|c|}{ 2. Time effect } & \\
\hline & & & & & & & F value & $P$ value & \\
\hline Hand massage (ml) & 3046.7 & \pm & 217.9 & 3008.3 & \pm & 211.8 & 0.16 & 0.70 & \\
\hline Machine massage (ml) & 3055.0 & \pm & 181.2 & 3009.4 & \pm & 182.4 & 26.19 & 0.00 & * \\
\hline
\end{tabular}

\begin{tabular}{|c|c|c|c|c|c|c|c|c|}
\hline & \multicolumn{3}{|c|}{ Hand massage } & \multicolumn{3}{|c|}{ Machine massage } & \multicolumn{2}{|c|}{$P$ value } \\
\hline & Mean & & SD & Mean & & SD & & \\
\hline Pleasant (point) & 4.5 & \pm & 0.6 & 3.6 & \pm & 0.6 & 0.00 & * \\
\hline Relaxation (point) & 4.1 & \pm & 0.9 & 3.4 & \pm & 0.8 & 0.00 & * \\
\hline Refreshing (point) & 4.1 & \pm & 0.8 & 3.3 & \pm & 0.8 & 0.03 & * \\
\hline
\end{tabular}

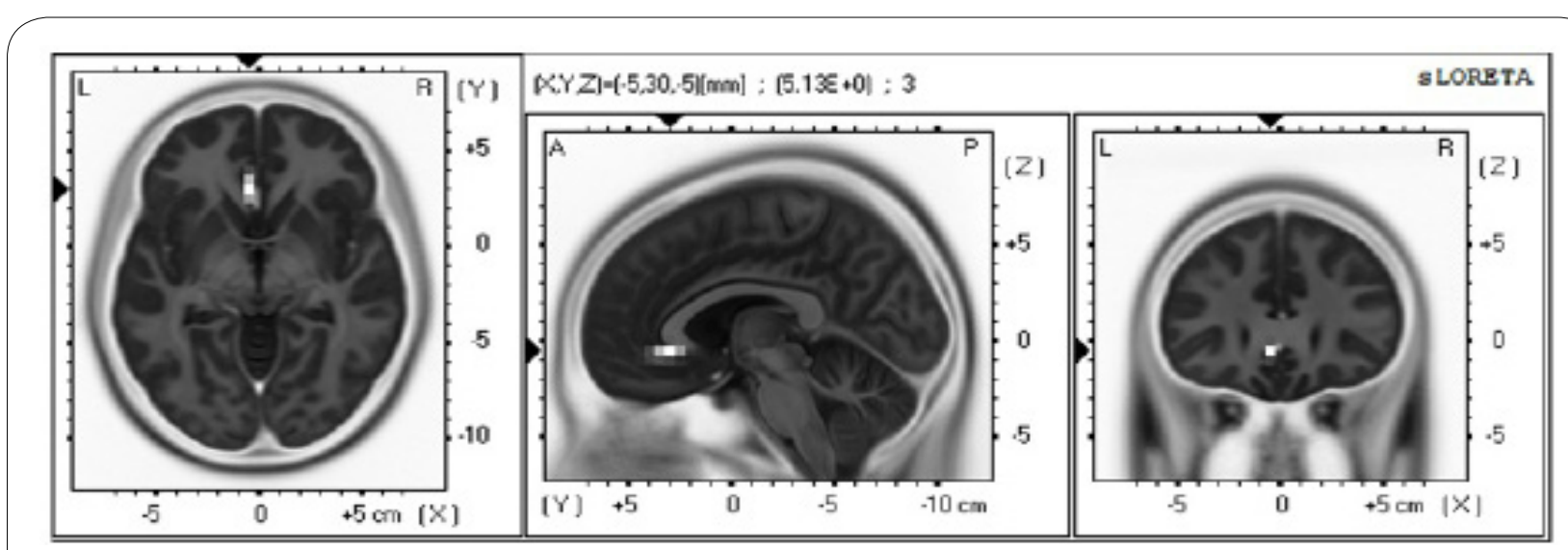

Figure 2: Statistical nonparametric maps (SnPM) of exact low- resolution brain electromagnetic tomography (eLORETA) of the alpha band comparing the post-hand and post-machine massages. The white color indicates increased activity after the post-hand massage. Images depicting the SnPM from different perspectives are based on voxel-by-voxel $t$-values of the differences. We found significantly increased alpha band activity in the left anterior cingulate cortex after hand massage $(\mathrm{p}<0.05)$, when compared with after machine massage.

\begin{tabular}{|l|l|l|l|}
\hline Brain region & Brodmann area & $\begin{array}{l}\text { MNI cords } \\
(\mathrm{x}, \mathrm{y}, \mathrm{z})\end{array}$ & P value \\
\hline Anterior cingulate cortex & 24 & $-5,30,-5$ & $<0.05$ \\
\hline Anterior cingulate cortex & 32 & $-5,35,-5$ & $<0.05$ \\
\hline
\end{tabular}

Table 3: Brain regionsexhibiting significantly higher activation of the alpha band after a hand massage than after a machine massage.

MNI, Montreal Neurological Institute 
Citation: Nakano H, Kodama T, Sakamoto M, Ueda T, Tani T, et al. (2018) Effect of Hand Massage on Occupational Leg Swelling and Resting-state Electroencephalographic Activity: A Randomized Cross-over Study. Int J Clin Res Trials 3: 125. doi: https://doi.org/10.15344/2456-8007/2018/125

Page 4 of 5

\section{Discussion}

This study aimed to investigate the effect of hand massage on occupational leg swelling and resting-state electroencephalographic (EEG) activity. We observed that occupational leg swelling was significantly reduced after hand and machine massages. It has been reported that compression stocking [1,4,5-18], neuromuscular electrical stimulation device [8] and leg movement $[9,10]$ are effective in improving occupational leg swelling. The present study suggests that the hand and machine massages could contribute as an additional tool to improve occupational leg swelling.

Partsch $\mathrm{H}$ et al., demonstrated that occupational leg edema was reduced $34.1 \mathrm{ml}$ using compression class A stocking (pressure at ankle level, $11.2 \mathrm{mmHg}$ ), $39.6 \mathrm{ml}$ using compression class I stocking (pressure at ankle level, $18.1 \mathrm{mmHg}$ ), and $59.1 \mathrm{ml}$ using compression class II stocking (pressure at ankle level, $21.8 \mathrm{mmHg}$ ) [5]. In another study, Mosti $\mathrm{G}$ et al., also reported that occupational leg edema was reduced by $40 \mathrm{ml}$ by using graduated elastic compression stockings (pressure at ankle level: sitting $=22 \mathrm{mmHg}$, standing $=25 \mathrm{mmHg}$; pressure at calf level: sitting $=18 \mathrm{mmHg}$, standing $=21 \mathrm{mmHg}$ ), and by $20 \mathrm{ml}$ using progressive elastic compression stockings (pressure at ankle level: sitting $=18 \mathrm{mmHg}$, standing $=19 \mathrm{mmHg}$; pressure at calf level: sitting $=30 \mathrm{mmHg}$, standing $=32.5 \mathrm{mmHg}$ ) [6]. On the other hand, occupational leg volume in this study was reduced by hand massage $(38.3 \mathrm{ml})$, and by machine massage $(45.6 \mathrm{ml})$. Thus, the reduction in occupational leg swelling observed in our study was similar to that observed in other studies.

The resting-state alpha activity, after hand massage, was significantly increased in the left anterior cingulate cortex compared with the resting-state alpha activity after machine massage. A previous study reported that movement performed with a human hand has a higher score of pleasantness and increases activity in the anterior cingulate cortex, when compared to movement with a rubber-gloved hand [29] In this study, the degree of pleasant was significantly higher after a hand massage than after a machine massage. Therefore, it is suggested that a hand massage is more pleasant than a machine massage, resulting in an increase of resting-state alpha activity in the anterior cingulate cortex. Additionally, since we observed lateralization in the left anterior cingulate cortex, the involvement of emotion and mood processing in the left frontal region may be considered. This is supported by a previous study that revealed an association between positive information / emotional processing and an increase in alpha activity in the left frontal region [30]. The present study revealed that hand massage increased the alpha activity in the left frontal region related to positive information / emotional processing because the degree of pleasant, relaxation and refreshing was significantly higher after a hand massage than after a machine massage. In addition, since the alpha activity in the frontal region reflects the activity of the anterior cingulate cortex [31], the present study identified the left anterior cingulate cortex as the source for the alpha activity, in the left frontal region and related to positive information / emotional processing, using the eLORETA analysis.

The present study does have a few limitations. First, since the hand massage was carried out with only one therapist, it is not certain whether other therapists will perform similar results. Second, longterm effects of hand massage and its effects on participants with any disease were not verified. Future studies are needed to verify the effect of hand massage taking into consideration the above issues.

\section{Conclusions}

This study investigated the effects of hand massage on occupational leg swelling and the resting-stage EEG activity. Results revealed that the lower leg volume was significantly reduced after hand and machine massages. Moreover, resting-state alpha activity after hand massage was significantly increased in the left anterior cingulate cortex compared with resting alpha activity after machine massage. This study suggests that hand massage improves occupational leg swelling and increases the resting-state alpha activity in the left anterior cingulate cortex.

\section{Competing Interests}

The authors declare that they have no competing interests.

\section{Author Contributions}

HN, TK and SM conceived and designed the experiments. HN, TK, MS, TU, TT, IM and SM performed the experiments. HN, TK, MS, TU and SM analyzed the data. HN, TK and SM wrote the paper.

\section{Acknowledgements}

We would like to thank all volunteers who participated in this study.

\section{References}

1. Blättler W, Kreis N, Lun B, Winiger J, Amsler F, et al. (2008) Leg symptoms of healthy people and their treatment with compression hosiery. Phlebology 23: 214-221.

2. Sudol-Szopinska I, Panorska AK, Kozinski P, BlachowiakK (2007) WorkRelated Chronic Venous Disease in Office and Bakery Workers. Occupational Ergonomics 7: 125-137.

3. de Boer EM, Broekhuijsen RW, Nieboer C, Bezemer PD (1999) Lycra Support Tights: Are They Effective? Phlebology 14: 162-166

4. Jungbeck C, Peterson K, Danielsson G, Norgren L (2002) Effects of Compression Hosiery in Female Workers with a Standing Profession. Phlebology 16: 117-120.

5. Partsch H, Winiger J, Lun B (2004) Compression stockings reduce occupational leg swelling. DermatolSurg 30: 737-743.

6. Mosti G, Partsch H (2013) Occupational leg oedema is more reduced by antigraduated than by graduated stockings. Eur J Vasc EndovascSurg 45: 523-527.

7. Blazek C, Amsler F, Blaettler W, Keo HH, Baumgartner I, et al. (2013) Compression hosiery for occupational leg symptoms and leg volume: a randomized crossover trial in a cohort of hairdressers. Phlebology 28: 239247.

8. Wou J, Williams KJ, Davies AH (2016) Compression Stockings versus Neuromuscular Electrical Stimulation Devices in the Management of Occupational Leg Swelling. Int J Angiol 25: 104-109.

9. Uda S, Seo A, Yoshinaga F (1997) Swell-preventing effect of intermittent exercise on lower leg during standing work. Ind Health 35: 36-40.

10. Lin $\mathrm{YH}, \mathrm{Chen} \mathrm{CY}, \mathrm{Cho} \mathrm{MH}$ (2012) Effectiveness of leg movement in reducing leg swelling and discomfort in lower extremities. Appl Ergon 43: 1033-1037.

11. Zhang Q, Styf J, Ekström L, Holm AK (2014) Effects of electrical nerve stimulation on force generation, oxygenation and blood volume in muscles of the immobilized human leg. Scand J Clin Lab Invest 74: 369-377.

12. Tucker A, Maass A, Bain D, Chen LH, Azzam M, et al. (2010) Augmentation of venous, arterial and microvascular blood supply in the leg by isometric neuromuscular stimulation via the peroneal nerve. Int J Angiol 19: e31-37.

13. Williams KJ, Moore HM, Davies AH (2015) Haemodynamic changes with the use of neuromuscular electrical stimulation compared to intermittent pneumatic compression. Phlebology 30: 365-372. 
Citation: Nakano H, Kodama T, Sakamoto M, Ueda T, Tani T, et al. (2018) Effect of Hand Massage on Occupational Leg Swelling and Resting-state Electroencephalographic Activity: A Randomized Cross-over Study. Int J Clin Res Trials 3: 125. doi: https://doi.org/10.15344/2456-8007/2018/125

Page 5 of 5

14. Doherty $D$ (2008) The physiological effects of massage on individuals with lymphoedema/chronic oedema, and its role in the development of clinical techniques used in practice: a systematic review. JBI Libr Syst Rev 6: 1-17.

15. Pyke C (2010) Massage: a helping hand for people with chronic oedema and lymphoedema. Br J Community Nurs 15: S28-30.

16. Coban A, Sirin A (2010) Effect of foot massage to decrease physiological lower leg oedema in late pregnancy: a randomized controlled trial in Turkey. Int J Nurs Pract 16: 454-460.

17. Jung GS, Choi IR, Kang HY, Choi EY (2017) Effects of Meridian Acupressure Massage on Body Composition, Edema, Stress, and Fatigue in Postpartum Women. J Altern Complement Med 23: 787-793.

18. Hodgson NA, Lafferty D (2012) Reflexology versus Swedish Massage to Reduce Physiologic Stress and Pain and Improve Mood in Nursing Home Residents with Cancer: A Pilot Trial. Evid Based Complement AlternatMed 2012: 456897.

19. Moyle W, Cooke ML, Beattie E, Shum DH, O'Dwyer ST, et al. (2014) Foot massage versus quiet presence on agitation and mood in people with dementia: a randomized controlled trial. Int J Nurs Stud 51: 856-864

20. Rabe E, Pannier-Fischer F, Bromen K, Schuldt K, Stang A, et al. (2003) Bonner Venenstudie der Deutschen Gesellschaft für Phlebologie. Epidemiologische Untersuchung zur Frage der Häufigkeit und Ausprägung von chronischen Venenkrankheiten in der städtischen und ländlichen Wohnbevölkerung Phlebologie 32: 1-14

21. Wang MY, Tsai PS, Lee PH, Chang WY, Yang CM, et al. (2008) The efficacy of reflexology: systematic review. J Adv Nurs 62: 512-520.

22. Pasley JD, O'Connor PJ (2008) High day-to-day reliability in lower leg volume measured by water displacement. Eur J ApplPhysiol 103: 393-398.

23. Likert R (1932) A technique for the measurement of attitudes. Archives of Psychology 22: 1-55.

24. Delorme A, Makeig S (2004) EEGLAB: an open source toolbox for analysis of single-trial EEG dynamics including independent component analysis. J Neurosci Methods 134: 9-21.

25. Pascual-Marqui RD (2007) Discrete, 3D distributed, linear imaging methods of electric neuronal activity. Part 1: exact, zero error localization. arXiv: 0710.3341: 1-16.

26. Fuchs M, Kastner J, Wagner M, Hawes S, Ebersole JS, et al. (2002) A standardized boundary element method volume conductor model. Clin Neurophysiol 113: 702-712.

27. Mazziotta J, Toga A, Evans A, Fox P, Lancaster J, et al. (2001) A probabilistic atlas and reference system for the human brain: International Consortium for Brain Mapping (ICBM). Philos Trans R SocLond B BiolSci 356: 1293-1322.

28. Crabbe JB, Dishman RK (2004) Brain electrocortical activity during and after exercise: a quantitative synthesis. Psychophysiology 41: 563-574

29. Lindgren L, Westling G, Brulin C, Lehtipalo S, Andersson M, et al. (2012) Pleasant human touch is represented in pregenual anterior cingulate cortex. Neuroimage 59: 3427-3432.

30. Coan JA, Allen JJ (2004) Frontal EEG asymmetry as a moderator and mediator of emotion. Biol Psychol 67: 7-49.

31. Yamamoto $S$, Kitamura $Y$, Yamada $N$, Nakashima $Y$, Kuroda $S$, et al (2006) Medial profrontal cortex and anterior cingulate cortex in the generation of alpha activity induced by transcendental meditation: a magnetoencephalographic study. Acta Med Okayama 60: 51-58. 